\title{
Wireless/Wired Automatic Switched Plasma Tweeter \& Speaker System
}

\author{
Rupali Singh $^{1}$, Yash Srivastava ${ }^{2}$, Prakhar Khare ${ }^{3}$, Tanuj Gupta ${ }^{4}$ \\ ECE, SRM University, India
}

\begin{abstract}
A Plasma Tweeter is an audio device which uses a pair of electrodes as a source of sound. It has a clear reproduction and Omni directional radiation pattern. A plasma tweeter has a better frequency response than a conventional speaker and does not involve any moving part (diaphragm) and thus has less reverberation and no wear and tear. Plasma tweeters invented earlier were very expensive. This paper presents a plasma audio system which is making the regular audio system more efficient because of the use of the latest plasma tweeter. Here the objective is to create a low cost and more efficient version of the most speakers invented till now with the complete audio system.
\end{abstract}

Keywords: Plasma, speaker, corona discharge.

\section{Introduction}

A plasma tweeter converts electrical signal into audio signal. It is done through the process of corona discharge. Unlike conventional speaker which uses diaphragm or cones as transducers, Plasma speaker uses discharge as a transducer. Plasma speaker have excellent high frequency response as they do not have any moving mechanical parts (like diaphragm) which limit the frequency response. Thus it has a clear reproduction and Omni directional radiation pattern but has a limited low frequency audio response. The first plasma speaker invented used flame for achieving the required thermal excitation [1]. To achieve the plasma arc a circuit acting as a pulse generator is stepped up using a flyback transformer which has two electrodes connected to its output. The drastic change in the input of the transformer creates high voltage through the secondary coil which is enough to create a plasma arc [2].This is equivalent to applying a certain change in energy $\Delta \mathrm{U}$ of the air between the electrodes. The air ionized is mostly atmospheric nitrogen and oxygen diatomic molecules. So,

$$
\Delta \mathrm{T}=(2 \Delta \mathrm{U}) / 3 \mathrm{R}
$$

Where

$\Delta \mathrm{T}=$ change in temperature of the gas

$\mathrm{R}=$ ideal gas constant

Considering the volume of the cylinder of the air getting ionized to be constant and applying gas Law,

Applying (1) in (2) we get

$$
\Delta \mathrm{P}=(\mathrm{nR} \Delta \mathrm{T}) / \mathrm{V}
$$

$$
\Delta \mathrm{P}=(2 \mathrm{n} \Delta \mathrm{U}) / 3 \mathrm{~V}
$$

Thus if we know the input energy we can know the varying pressure and can tune accordingly for the desired output. The audio signal is modulated with the square wave creating PWM which is fed to flyback. The frequency of the square wave is above $20 \mathrm{kHz}$ and thus cannot be heard by human ear and thus only the variations caused by the audio wave in PWM output can be heard by the human ear.

\section{Block Diagram}

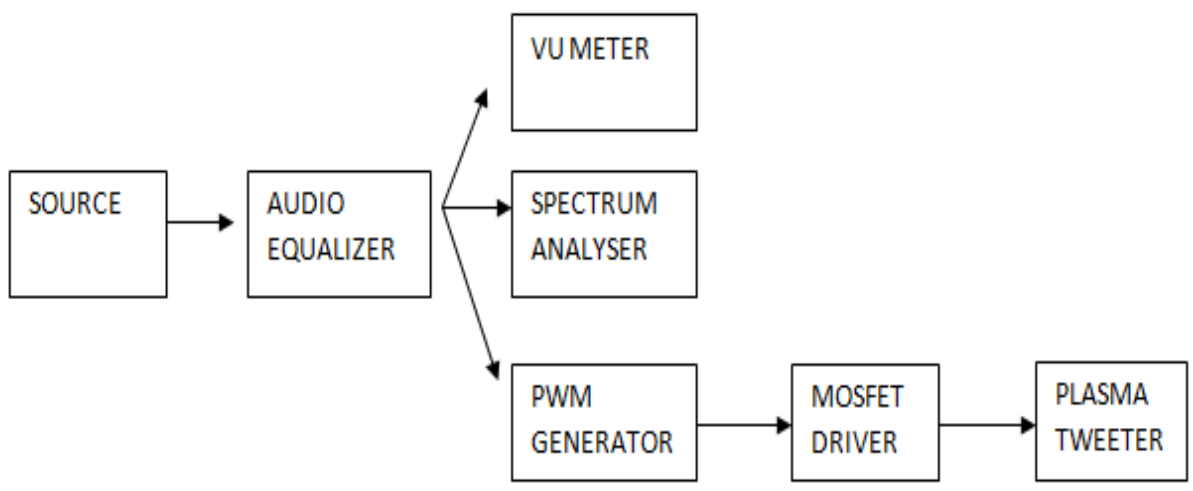

Figure 1 (Complete System) 
The source can be either wireless or wired. The wireless source includes FM and Infrared. For wired input AUX cable is used for this purpose. There is separate input for Bluetooth. After the input is given the message signal (audio) passes through the audio equalizer which is used for correction of any deficiencies in the linearity of speaker system or living room. After passing through the equalizer the message passes through the VU (volume unit). It displays the signal level in the audio device. Simultaneously it passes through the Spectrum analyzer and PWM generator. Spectrum analyzer measures the magnitude of the Input signal versus the frequency. PWM generator modulates the input signal with the square wave of high frequency which drives the MOSFET driver and thus finally producing the corona discharge giving audio output.

Below shown figure is the complete setup for the Full bridge flyback driver without feedback, driven by a fixed frequency and an audio modulated duty cycle.

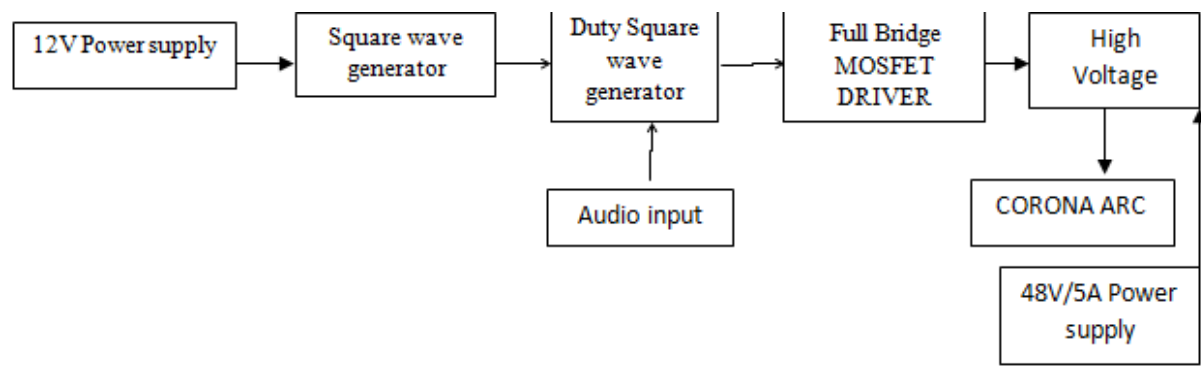

Figure 2 (Plasma setup without feedback)

Second figure shows the feedback driven square wave generator via which flyback is driven making a closed loop resonant mode of flyback producing a brighter arc.

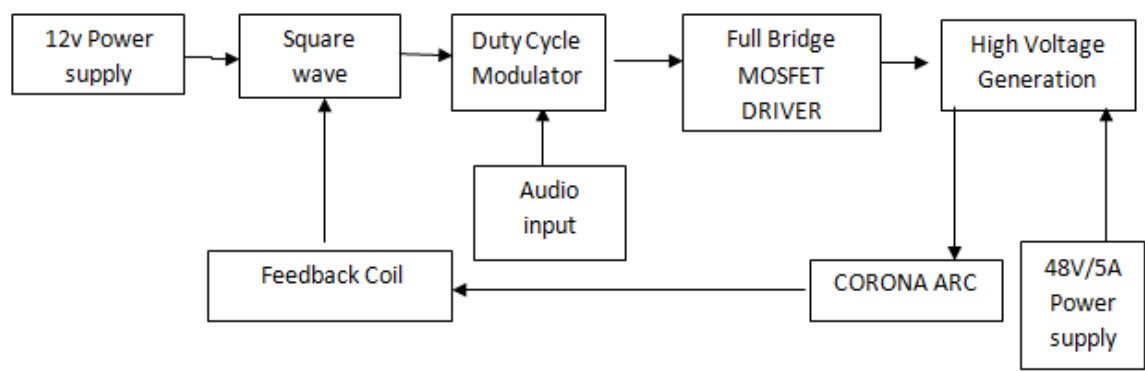

Figure 3 (Feedback switched plasma tweeter)

The frequency is set between $22 \mathrm{KHz}$ to $100 \mathrm{KHz}$ however most of the flybacks have the resonance frequency between $18 \mathrm{KHz}$ up to $100 \mathrm{KHz}$ hence three cases arises:

1. Drive the flyback with a fixed Frequency (say $48 \mathrm{KHz}$ ).

2. Make a small Slayer exciter circuit using the feedback of the Flyback transformer and measure the frequency at the base of BJT (NOTE: The BJT should be mounted on a large Heat sink) and using this frequency to design a driver for flyback.

3. Due to change in environment the overall capacitance of the coil changes which is analogy of tesla coil. Hence a slight change in environment will cause the frequency to change and so design a driver that automatically drives the flyback on its resonance frequency and hence will be a universal flyback driver.

Full Bridge Circuit Using Ir2110

\section{Circuit Diagram And Overview}

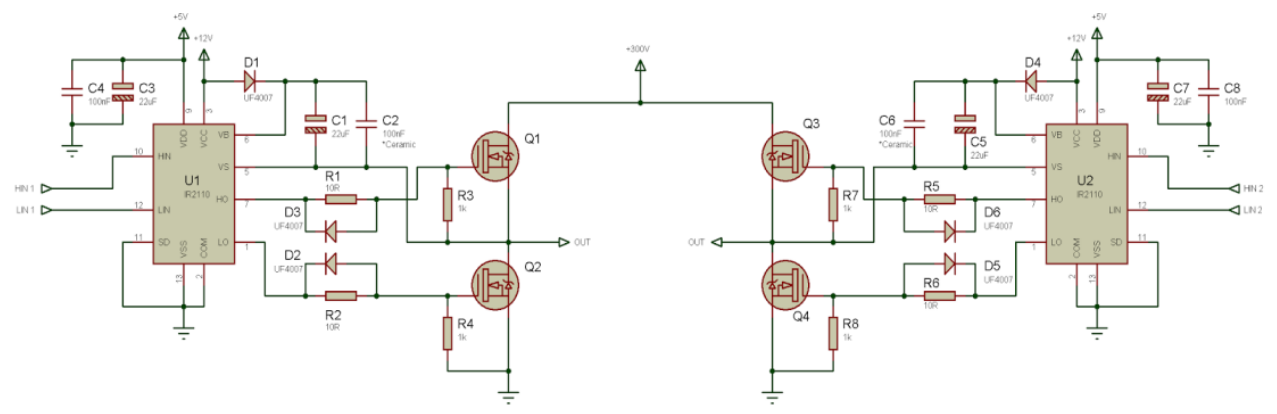

Figure 4 
During the turning on/off of the MOSFETs due to parasitic capacitance in circuit such as leakage capacitance, miller capacitance, gate capacitance of the MOSFET and its surroundings causes following two effects:-

- Firstly, the improper turning on or off due to gate capacitance. Since it is hard to discharge and instantly turn it off. Hence using a MOSFET driver (Half Bridge Driver in A Full Bridge [3] configuration) will allow the use of MOSFET during the both positive square wave and negative square wave .Hence preventing heating of MOSFETs which was in case of single ended MOSFET driven flyback circuit since in that circuit, when the FET is off whole voltage is dissipated around the internal power diode which causes heating when operated at high voltages at higher frequencies. Full Bridge allows us to operate at slight higher voltages and high current without causing heating failure of MOSFETs.

- Secondly, the inductance of the coil with the capacitance of MOSFET and parasitic capacitance causes Ringing effect or oscillations in every pulse .The turning-off of the power switch interrupts the current through the leakage inductance of the transformer, which causes a voltage spike on the drain of the FET .The inductance will then ring with stray capacitances in the circuit, producing large amplitude highfrequency waveforms.

The Complete Circuit diagram is shown in below figure:

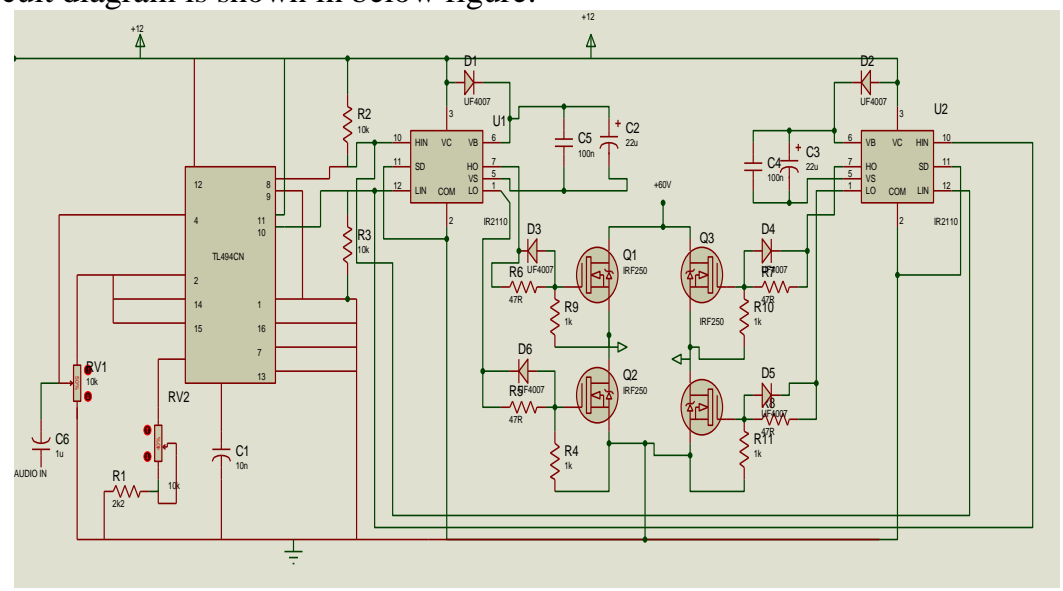

Figure 5

The two output BJTs (internally built) are configured as common emitter and emitter follower configuration and hence two inverted pulses are observed. Two pulses are fed in HI (High input) and LI(Low Input)for one bridge IC and for the next IC the two signals will be fed in reverse order that is the HI signal for the first IC will also be fed on the second IC but at LI pin and vice versa.This circuit lacks the Snubber circuit which is needed to suppress the voltage spikes that can kill the MOSFET. Since just by powering the circuit with a $12 \mathrm{~V}$ supply can cause a back EMF spike of about 100 volts and hence the MOSFET chosen is rated for 200V/30A. This is the effect commonly known as EMF kick back or FLYBACK effect. A simple dissipative RCD snubber can be designed and used or a non dissipative LC snubber can be designed for this particular setup which will reduce the ringing effect or in simple words it will snap back the output waveform to absolute rail voltages.

\section{Audio Equalizer}

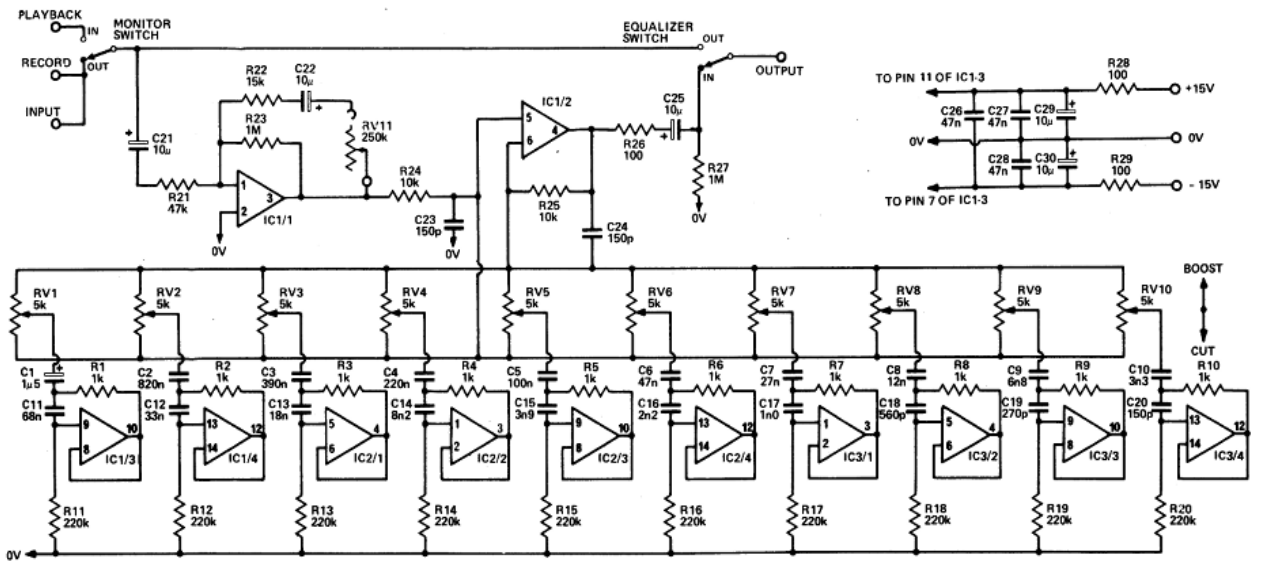

Figure 6 
Audio graphic equalizer [4] circuit is used to achieve equalization in sound as per need. It is a process of adjusting balance between the frequency components within an electronic signal. The circuit of graphic audio equalizer, allocates ten adjusting potentiometer, that each one from them affects in a predetermined area of frequencies, the central frequency of which abstains a octave, from the central frequencies of neighboring regions. The equalizer used is a 10 band equalizer. In this 10 audio band pass filters are used for the purpose of separating different frequencies of audio. Various operational amplifiers has been used for the same purpose. For this purpose IC TL 074 has been used which compromises of 4 inbuilt operational amplifiers. Each op-amp used has its own output. There are inverting and non inverting pins for each op-amp. The pin used for providing power is 4 as Vcc+ and 11 for Vcc-. The power supply used for this circuit is symmetrical (dual polarity) type, 15V DC. This circuit must be connected before the amplifier circuit. With good components and good power supply, the sound quality will be better. This is useful in removing the unwanted signal or to make certain voices more prominent.

There are 10 Audio band pass filters whose frequency can be easily calculated using the standard formula $\mathrm{F}=1 / 2 *$ pi $*$ sqrt $(\mathrm{R} 1 * \mathrm{C} 1 * \mathrm{R} 2 * \mathrm{C} 2)$

The frequency ranges from $32 \mathrm{~Hz}$ up to $16 \mathrm{KHz}$.theoretical values and practical values will differ greatly due to unavailability of the calculated value parts. All those Band pass filters are also amplifiers whose gain can be set low to high according to need and the preamplifier is provided to amplify the sound further.

\section{Preamplifier And 60 Watt Amplifier}

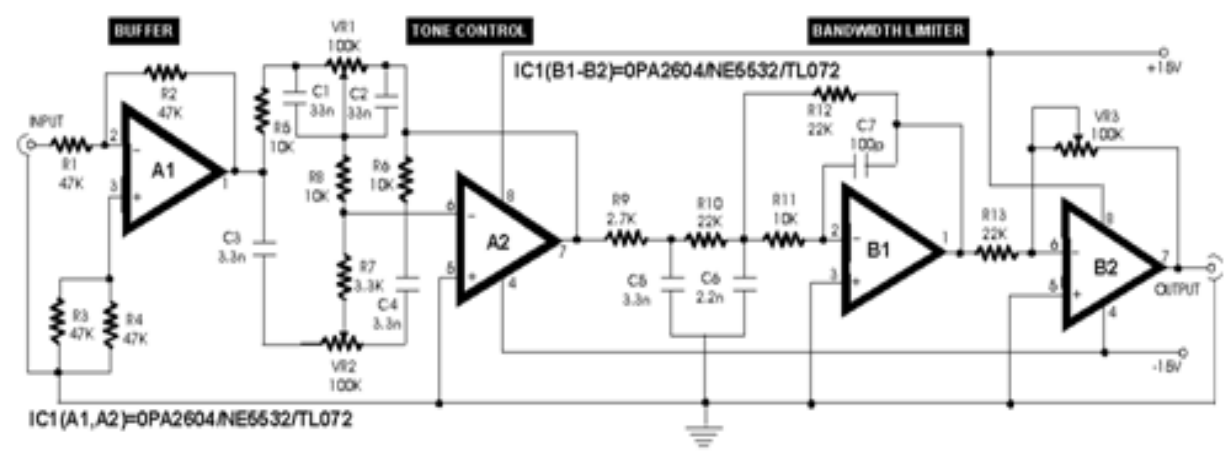

Figure 7 (Preamplifier)

Preamplifier is used for amplifying the signal before it is sent to main audio system. It is done to increase the voltage level of the low power audio signal. This circuit is divided in four parts namely Buffer, Tone control, Bandwidth limiter, output amplifier. The buffer is used to isolate the audio signal from the source to the main system i.e passing the audio signal from the source to the main system without actually connecting the signal to system directly. Tone control circuit is used to control the tone of the signal i.e to increase or decrease the Bass $(<500 \mathrm{~Hz})$, Treble $(>1 \mathrm{KHz})$ while swinging between the middle tone simultaneously. Bandwidth limiter limits the bandwidth for avoiding the high frequency noises. Lastly, the amplifier is used to increase overall gain of the output i.e $\{$ gain=4.5(Max) to 0.045 (Min) $\}$.

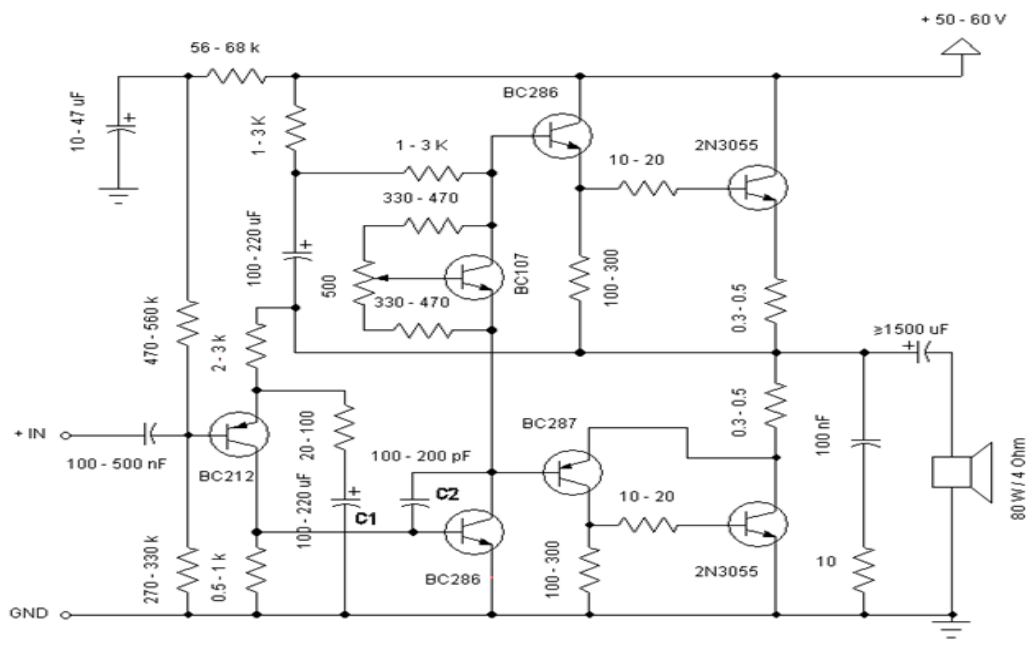

Figure 8 (Audio Power Amplifier) 
The Power amplifier is used to increase the voltage as well as the current of the output audio signal. Wide ranges of resistors and capacitors allow us to work on wide range of input voltages.

\section{Spectrum Analyzer}

A spectrum analyzer [5] is a device that is used technically for assessing a frequency source with respect to its strength. It is used in getting a visual display to show the different bands. It measures the magnitude of an input signal versus frequency within the full frequency range of the instrument. The primary use is to measure the power of the spectrum of known and unknown signals. The input signals that a spectrum analyzer measures is electrical; however, spectral compositions of other signals, such as acoustic pressure waves and optical light waves, can be considered through the use of an appropriate transducer. It can be analyzed that the different components of a signal are not easily detectable in time domain waveforms. These parameters are useful in the characterization of electronic devices, such as wireless transmitters. This circuit is embedded with the plasma tweeter circuit and also can be used as a standalone circuit. It can display the real time graph spectrogram on a LED display which has 100 LED's. It produces very nice visual effects and simultaneously provides an effective observable way to inspect audio performance during playback, recording, or any tonal adjustment (bass, treble, mid-frequency adjustment, or adaptive equalization) on equalizer.

It Consist of Preamplifier $\rightarrow 10 x$ Band Pass Filters $\rightarrow$ Peak Detector $\rightarrow 10$ Bilateral switches $\rightarrow 20$ Switching BJTs $\rightarrow 10$ Volume Comparators $\rightarrow 100$ LEDs (for display)

For the Preamplifier, Band pass Filters, comparators TL074 IC is used (Quad OPAMP) And for the Switching action CD4066 is used (QUAD BILATERAL SWITCH). CD4017 Decade counter activates all the switches one by one hence multiplexes the signal on a single line to compare.

\section{Fm/Infrared And Its Switching}

In Infrared[6]

Audio signal $\rightarrow 566$ VCO Modulator (38Khz Osc Freq) $\rightarrow$ Drive 2N3904 and 3906 BJTs $\rightarrow$ Infrared LEDs

Photosignal $\rightarrow$ IR Detector $(1838 \mathrm{~T})(38 \mathrm{Khz}$ IR detector with inbuilt Amplifier and noise reduction technology) $\rightarrow$ Inverting Buffer +565 PLL $\rightarrow$ Amplifier $\rightarrow$ Sound Out

(same) BUFFER $\rightarrow$ comparator to see if voltage is there or not if not then switch to FM.

Since the audio is modulated over a $38 \mathrm{Khz}$ signal using a vco there is no distortion or interfernce of nearby lights and the PLL can easily detect it as FM signal and can extract the signal easily.

Switching

CD4066 IC is used for switching, giving priority to the infrared and then switchng for FM when there is obstruction.

\section{Results And Discussion}

After so many circuit design studies and implementation we came to following conclusions:

- For the Audio Modulated Plasma Arc non rectified flyback transformer are better than rectified flyback transformers. Modern CRT TV flybacks are rectified flyback transformer with the High Voltage diode inbuilt in it with some capacitors.

- Full Bridge is more efficient than single ended mosfet driven flyback transformer circuit. Meaning more input voltage and current can be fed into it.

- Ringing is introduced in the circuit but is reduced with a LC snubber[7] made in parallel with primary winding designed with usual hit and error method since parasitic capacitance is not known and reduced the distortion as well as spikes from output to much extent.

- Circuit works best with IR2110 or with IRS2104 rather than TC4420/4429 with t1494 and IRFP250N Pairs of MOSFET.

- Audio can be given on both potentiometer of the Timing resistor or the dead time resistor but input at the timing resistor will give you Frequency Modulated Signal while input at the dead time resistor will give you Pulse Width Modulated Output when tested with $8 \mathrm{Ohm}$ Speaker the PWM was Cleaner while FM was much heavily distorted.

- Most of the Flybacks are Rated for $110 \mathrm{~V}$ at primary since the flyback used in circuit is rated for $24 \mathrm{Kv}$ at secondary with 50volts input we achieved approx $5 \mathrm{~cm}$ arcs.

- Increasing Voltage at Primary increase spark length and hence the Audio Volume from arc while more Current will increase the temperature of arc causing more air molecules to vibrate due to heat and obtain energy faster increases frequency response up to lower frequencies than $1 \mathrm{KHz}$ increases Bass response.

- Audio Spectrum analyzer although the ICs 4016/4066 are CMOS based ICs but are used to switch analog signals and are the only analog ICs among the Digital CMOS family of 4000 series. 
- Spectrum Analyser circuit is Huge since we made it in old-school ANALOG style but it requires no Microprocessor or modern circuitry to perform .although the clock part and the flash comparator have a modern substitute as NE555 and LM3915 Respectively.

- $\quad$ FM and Infrared Circuitry were not that easy to build and the circuits available on sources need to be simulated before actual building.

\section{Conclusions}

After testing many circuits, it has been found that full bridge circuit works best for plasma tweeter. Flyback has been used at 50V without any heating failure. By using Snubber circuit the input voltage has been raised to $90 \%$ of the total rated input voltage. Thus high frequency response is achieved with good audio output. Till now the circuits are driven at fixed frequency but in future universal flyback driver can be made so that any flyback transformer can be driven automatically. All flyback transformer works mostly above 1 KHZ but a transformer can be made so that it can work on high voltage with low excitation frequency needed to operate it. Replacing flyback with Tesla coil energy can be transferred wirelessly from the audio modulated coil to the receiver coil to glow LED's or play music. Once the base response is increased low cost plasma tweeter can replace the costly conventional speakers.

\section{References}

[1]. Electronic Refereed Journal Article, MUSICAL FLAMES, W.R. Babcock, K.L. Baker and Cattaneo.A.G, Nature Volume 216, Issue 5116, pp.676-678(1967) DOI: 10.1038/216676b0

[2]. An Improved Winding Loss Analytical Model of Flyback Transformer, Wei Yuan, Xiucheng Huang, Peipei Meng, Guoxing Zhang Published in: Applied Power Electronics Conference and Exposition (APEC), 2010 Twenty fifth Annual IEEE DOI: 10.1109/APEC.2010.5433633

[3]. Construction of Full-Bridge Stereo Plasma Speaker, Dan Peterson \& Gino Giannetti, PHYS 406 Final Paper, May 13th, 2016

[4]. "High-Precision Parallel Graphic Equalizer"- Published in: IEEE/ACM Transactions on Audio, Speech, and Language Processing (Volume: 22, Issue: 12, Dec. 2014 ),DOI: 10.1109/TASLP.2014.2354241 ,Page(s): $1894-1904$.

[5]. Matthew T. Hunter, Achilleas G. Kourtellis, Christopher D. Ziomek, Wasfy B. Mikhael "Fundamentals of Modern Spectral Analysis "IEEE,978-1-4244-7959-7/10,2010.

[6]. Development of Optical Wireless Audio System Using Infrared Light Communications:-IOSR Journal of Electronics and Communication Engineering (IOSR-JECE) e-ISSN: 2278-2834,p- ISSN: 2278-8735.Volume 8, Issue 6 (Nov. - Dec. 2013), PP 65-69

[7]. OPTIMUM SNUBBERS FOR POWER SEMICONDUCTORS, by William McMurray, IEEE IAS Transactions, Vol. IA-8, No. 5, Sept/Oct 1972, pp. 593-600. 